\title{
ATUAÇÃO DA ENFERMAGEM NA CLASSIFICAÇÃO DE RISCO DO SERVIÇO DE URGÊNCIA EMERGÊNCIA
}

\author{
Ely de Almeida Glória Filho ${ }^{1}$ \\ Matheus Coutinho Clen Sodré ${ }^{2}$
}

RESUMO: As urgências e emergências acaba sendo uma das portas de entradas dos usuários no SUS, o que na maioria das vezes, resulta em superlotação devido a excessiva demanda. O estudo trata-se de revisão integrativa com o objetivo de conhecer e avaliar a implantação da classificação de risco no serviço de urgência e emergência. A crescente demanda por serviços nesta área nos últimos anos, devida ao crescimento do número de acidentes e da violência urbana e a insuficiência estruturação da rede são fatores que têm contribuído decisivamente para a sobrecarga de serviços de urgência e emergência disponibilizados para o atendimento da população. Em 2004 o Ministério da Saúde fez uma reestruturação na urgência e emergência, implementando o acolhimento com classificação de risco que objetiva a reorganização a atenção, tendo em vista o acolhimento ao usuário e a priorização do atendimento, de acordo com a gravidade do risco ou quadro apresentado, para então começar a sistematização do atendimento para que seja com mais agilidade, segurança e humanização. Esta é uma pesquisa de revisão integrativa que é um método que reúne os resultados obtidos sobre um mesmo assunto, e tem por finalidade sintetizar e analisar os dados para desenvolver uma explicação mais abrangente de um assunto em específico.

Palavras-chaves: Classificação de risco. Acolhimento. Urgência e emergência.

ABSTRACT: Urges and emergencies end up being one of the gateways for users to the SUS, which most often results in overcrowding due to excessive demand. The study is a field research with the objective of knowing and evaluating the implementation of risk classification in the urgent and emergency service. The growing demand for services in this area in recent years, due to the increase in the number of accidents and urban violence and the insufficiency of the network's structure, are factors that have contributed decisively to the overload of urgent and emergency services. In 2004, the Ministry of Health carried out an urgency and emergency, implementing care with risk classification that aims to reorganize care, with a view to welcoming the user and prioritizing care, according to the severity of the risk or situation presented, to then start systematizing the service so that it is

\footnotetext{
${ }^{I}$ Bacharelado em Enfermagem- UniRedentor Centro Universitário. E-mail: elyfilho98@hotmail.com.

${ }^{2}$ Bacharelado em Enfermagem _ UniRedentor Centro Universitário.
} 
more agile, safe and humane. This is an integrative review research that is a method that gathers the results obtained on the same subject, and has to develop, synthesize and analyze the data to develop a more specific explanation of a specific subject.

Keywords: Risk classification. Reception. Urgency and emergency.

\section{INTRODUÇÃO}

Os serviços de urgência e emergência são feitos para atender qualquer tipo de agravo a saúde, funcionando como um serviço de saúde independente ou inserido em um estabelecimento de internação com maior capacidade de atendimento.

O enfermeiro que atua na triagem é um protagonista no acolhimento com classificação de risco de Manchester. É imprescindível que este profissional reflita sobre o desafio de utilizar este tipo de ferramenta tecnológica e esteja sempre se aprimorando para garantir a eficácia e resolutividade operacional para a assistência de maneira dinâmica e habilidosa. O processo de triagem com classificação de risco se consolida como uma reorganização do trabalho coletivo de equipe e se torna um processo de inovação e mudança para acolher o usuário. (ULHOA et al 2009)

As ações do enfermeiro em unidades de urgência e emergência estão diretamente relacionadas à necessidade do paciente, objetivando a melhora do seu quadro clínico e prevenção de agravos à saúde. (SOUZA \& CHAGAS, 2018)

A área de Urgência e Emergência constitui-se em um importante componente da assistência à saúde. A crescente demanda por serviços nesta área nos últimos anos, devida ao crescimento do número de acidentes e da violência urbana e a insuficiente estruturação da rede são fatores que têm contribuído decisivamente para a sobrecarga de serviços de Urgência e Emergência disponibilizados para o atendimento da população. Isso tem transformado esta área numa das mais problemáticas do Sistema de Saúde. (BRASIL.MINISTÉRIO DA SAÚDE 2002).

Com todo o crescimento de demanda que a urgência e emergência vem sofrendo nos últimos anos, devemos entender a atenção básica a saúde, que é a porta de entrada a assistência em saúde. Essa rede de atenção tem como objetivo a ampliação do acesso, o fortalecimento do vínculo, a responsabilização e o primeiro atendimento as urgência e emergência em ambiente adequado, até a transferência dos pacientes a outros pontos de atenção. Esses objetivos não sendo realizados na prática, 
a urgência e emergência tem grandes consequências de ter grandes lotações e acabando não atendendo as devidas demandas.

Para entender melhor o Sistemas Estaduais de Urgência e Emergência a portaria 2.048 - 2002 define:

Assim, torna-se imperativo estruturar os Sistemas Estaduais de Urgência e Emergência de forma a envolver toda a rede assistencial, desde a rede pré-hospitalar, (unidades básicas de saúde, programa de saúde da família (PSF), ambulatórios especializados, serviços de diagnóstico e terapias, unidades não hospitalares), serviços de atendimento pré-hospitalar móvel (SAMU, Resgate, ambulâncias do setor privado, etc.), até a rede hospitalar de alta complexidade, capacitando e responsabilizando cada um destes componentes da rede assistencial pela atenção a uma determinada parcela da demanda de urgência, respeitados os limites de sua complexidade e capacidade de resolução.

Em 2004 o ministério da saúde fez uma reestruturação na urgência e emergência, implementando o Acolhimento com Classificação e Avaliação de Risco que objetiva a reorganização a atenção, tendo em vista o acolhimento ao usuário e a priorização do atendimento, de acordo com a gravidade do risco ou quadro apresentado, para então começar a sistematização do atendimento para que seja com mais agilidade, segurança e humanização. Esse sistema colabora muito com os profissionais por poder controlar a demanda, otimizar o atendimento às urgências e emergências, diminuir a sobrecarga ocupacional da equipe de saúde e fortalecer o vínculo usuário-trabalhador.

O acolhimento é um modo de operar os processos de trabalho em saúde, de forma a atender a todos que procuram os serviços de saúde, ouvindo seus pedidos e assumindo no serviço uma postura capaz de acolher, escutar e dar respostas mais adequadas aos usuários. Ou seja, requer prestar um atendimento com resolutividade e responsabilização, orientando, quando for o caso, o paciente e a família em relação a outros serviços de saúde, para a continuidade da assistência, e estabelecendo articulações com esses serviços, para garantir a eficácia desses encaminhamentos. (BRASIL. MINISTÉRIO DA SAÚDE 2010) 
No atendimento pelo Acolhimento Com Classificação de Risco (ACCR), após o usuário ser acolhido no serviço, utiliza-se o ordenamento estratificado na forma de, no mínimo, quatro cores para classificar os agravos: vermelho (emergência); amarelo (urgência); verde (menor urgência); e azul (não urgência), representando, respectivamente, a escala do maior ao menor risco, segundo critérios clínicos estabelecidos por protocolos institucionalizados. Ainda, os serviços emergenciais podem adotar sistemas com outras categorias para o atendimento, ao exemplo do protocolo de Manchester, que é muito utilizado globalmente e acrescenta a cor laranja aos pacientes classificados como "muito urgentes", ou seja, um intermédio entre as cores vermelha e amarela antes citadas. (OLIVEIRA et al 2017)

Aplicação de protocolos proporciona o melhor desempenho e segurança do enfermeiro na classificação qualificada do usuário, com isso vira um apoio na tomada de decisões e na avaliação, tendo em vista a atitude e o conhecimento teórico e prático como habilidades imprescindíveis deste profissional. Sendo assim a pesquisa tem como objetivo avaliar a implantação de sistemas de classificação de risco como instrumento de organização da rede de urgência e emergência.

\section{MATERIAIS E MÉTODOS}

O estudo trata-se de uma revisão integrativa de pesquisa, que é um método que reúne os resultados obtidos de pesquisas sobre um mesmo assunto, e tem por finalidade sintetizar e analisar os dados para desenvolver uma explicação mais abrangente de um assunto em específico.

As etapas que conduziram esta revisão integrativa sobre o papel do enfermeiro na implantação da classificação de risco do serviço de urgência e emergência encontram-se descritas a seguir.

Inicialmente foi realizado levantamento de artigos no período de setembro/ outubro de 2021, sendo definidos os materiais que tinham relação com a pesquisa e que puderam ser acessados pelo pesquisador. Foram utilizadas pesquisas cadastradas nas bases de dados da Literatura Latino-Americano e do Caribe Ciências da Saúde (LILACS), Scientific Eletronic Library Online (SCIELO). 
Os descritores utilizados foram: Triagem, acolhimento com classificação de riscos, protocolo de Manchester e, urgência e emergência

Foi utilizado como critério de inclusão artigos com temas pertinentes à pesquisa publicados entre 2007 e 2021.

Como critério de exclusão, estudos cujo tema não atendiam ao objetivo do trabalho, que não estavam no idioma português Brasil, não publicados entre o período de 2007 a 2021 e artigos que não tinham acesso ao texto completo e não disponíveis ao online.

Para a avaliação de dados foi confeccionado um instrumento de coleta de dados, com o objetivo de organizar os dados coletados através da revisão integrativa, que compreendeu: dados de identificação (título do trabalho, autores, periódico, ano, volume, número, descritores/palavra chaves); objetivo/questão de investigação dos estudos; metodologia; resultados/aplicabilidade da classificação de risco pelo enfermeiro, limitações/recomendações; e conclusões.

Ocorreu a análise dos artigos, a partir de seus objetivos, metodologia empregada e resultados encontrados, sintetizando os resultados por categoria temática.

\section{RESULTADOS}

Nos quinze artigos utilizados nesse estudo, buscou-se conhecer como é importante a atuação do enfermeiro na classificação de risco do serviço de urgência e emergência. Com base no material consultado, constatou que a temática apresentou uma boa quantidade de artigos, que refletiu em uma seleção mais rigorosa de artigos que permite uma produção científica referente ao tema.

Foram encontrados 75 artigos nas bases de dados do LILCAS (66,6\%) e SciELO (33,3\%), com a utilização de todos os descritores. Após a leitura desses artigos foram excluídos 60 artigos (8o,0\%), com a utilização do critério de exclusão. Sendo assim, foram selecionados 15 artigos (20\%) como amostra final. 
Tabela I - Distribuição dos artigos localizados, excluídos e selecionado nas bases eletrônicas de dado - 2007-2021

\begin{tabular}{|l|l|l|l|}
\hline Base de dados & Localizados & Excluídos & Amostra final \\
\hline LILACS & $50(66,6 \%)$ & $42(84 \%)$ & $8(16,0 \%)$ \\
\hline SciELO & $25(33,3 \%)$ & $18(72 \%)$ & $7(28,0 \%)$ \\
\hline Total & $75(100 \%)$ & $60(80 \%)$ & $15(20,0 \%)$ \\
\hline
\end{tabular}

Houveram $7(46,6 \%)$ artigos voltados para ter um conhecimento mais aprofundado sobre o acolhimento com classificação de risco, 2 (13,3\%) sobre o protocolo de Manchester como um instrumento dos enfermeiros para a classificação de risco, 2 (13,3\%) voltados para a humanização e segurança do paciente nos serviços de urgência e emergência, e $4(26,6 \%)$ com foco em analisar a qualidade e demanda dos serviços de urgência emergência utilizadores do acolhimento com classificação de risco.

Para uma melhor análise e discussão dos dados com intuito de facilitar a compreensão dos resultados obtidos, demonstra-se a seguir a tabela com os artigos que foram analisados no estudo. A tabela apresenta artigos relevantes sobre a implantação da classificação de riscos direcionado pelo trabalho.

Tabela 2- Estudos selecionados por ano de publicação, títulos e periódicos 2007- 202I

\begin{tabular}{|l|l|l|l|}
\hline No & Ano & \multicolumn{1}{|c|}{ Título } & Periódicos \\
\hline I & 2007 & Triagem em serviços de saúde: percepções dos usuários & $\begin{array}{l}\text { Rev. Latino- } \\
\text { Am. Enf. }\end{array}$ \\
\hline 2 & 2021 & $\begin{array}{l}\text { Segurança do paciente em uma unidade de pronto atendimento: } \\
\text { planejamento de ações estratégicas }\end{array}$ & $\begin{array}{l}\text { Rev. Enf. } \\
\text { UERJ. }\end{array}$ \\
\hline 3 & 2013 & $\begin{array}{l}\text { Acolhimento com classificação de risco em hospitais de ensino: } \\
\text { avaliação da estrutura, processo e resultado }\end{array}$ & $\begin{array}{l}\text { Rev. Latino- } \\
\text { Am. Enf. }\end{array}$ \\
\hline 4 & 2018 & $\begin{array}{l}\text { Satisfação de usuários com o acolhimento e classificação de } \\
\text { risco em unidades públicas de saúde }\end{array}$ & $\begin{array}{l}\text { Rev. } \\
\text { Enf. }\end{array}$ \\
\hline 5 & 2018 & $\begin{array}{l}\text { Concotr. } \\
\text { Concordância de um protocolo institucional de avaliação com }\end{array}$ & \begin{tabular}{l} 
Texto \\
\hline
\end{tabular}
\end{tabular}




\begin{tabular}{|c|c|c|c|}
\hline & & classificação de risco & $\begin{array}{l}\text { contexto } \\
\text { Enferm. }\end{array}$ \\
\hline 6 & 2015 & $\begin{array}{l}\text { Análise da demanda atendida em unidade de urgência com } \\
\text { classificação de risco }\end{array}$ & $\begin{array}{l}\text { Saúde em } \\
\text { debate }\end{array}$ \\
\hline 7 & 2015 & $\begin{array}{l}\text { Acolhimento com Classificação de Risco: Avaliação de } \\
\text { Serviços Hospitalares de Emergência }\end{array}$ & $\begin{array}{l}\text { Esc. Anna } \\
\text { Nery }\end{array}$ \\
\hline 8 & 2015 & $\begin{array}{l}\text { Avaliação da qualidade da Classificação de Risco nos Serviços } \\
\text { de Emergência }\end{array}$ & $\begin{array}{l}\text { Acta Paul } \\
\text { Enferm. }\end{array}$ \\
\hline 9 & 2019 & $\begin{array}{l}\text { ANÁLISE DO ACOLHIMENTO COM CLASSIFICAÇÃO } \\
\text { DE RISCO EM UNIDADES DE PRONTO- } \\
\text { ATENDIMENTO }\end{array}$ & $\begin{array}{l}\text { Rev. } \quad \text { Min } \\
\text { Enferm. }\end{array}$ \\
\hline IO & 2015 & $\begin{array}{l}\text { Valor de predição do Sistema de Triagem de Manchester: } \\
\text { avaliação dos desfechos clínicos de pacientes }\end{array}$ & REBEn \\
\hline II & 2017 & $\begin{array}{l}\text { Protocolo de Manchester e população usuária na classificação } \\
\text { de risco: visão do enfermeiro }\end{array}$ & REB \\
\hline I2 & 2019 & $\begin{array}{l}\text { Humanização nos serviços de urgência e emergência: } \\
\text { contribuições para o cuidado de enfermagem }\end{array}$ & RGE \\
\hline 13 & 2019 & $\begin{array}{l}\text { A Atuação do Enfermeiro no Sistema de Acolhimento e } \\
\text { Classificação de Risco nos Serviços de Saúde }\end{array}$ & RGE \\
\hline I4 & 2018 & $\begin{array}{l}\text { Acolhimento com avaliação e classificação de risco em um } \\
\text { pronto socorro: estudo comparativo }\end{array}$ & ISSN \\
\hline 15 & 2017 & $\begin{array}{l}\text { Acolhimento com classificação de risco em unidade de pronto } \\
\text { atendimento: estudo avaliativo }\end{array}$ & $\begin{array}{l}\text { Rev Esc } \\
\text { Enferm USP }\end{array}$ \\
\hline
\end{tabular}

\section{DISCUSSÃO}

4.I O Protagonismo do Enfermeiro na realização do Acolhimento com Classificação de Risco

As unidades de serviço de urgência e emergência são destinadas ao atendimento de pacientes com problemas agudos e com alta gravidade, com garantia 
de assistência rápida e imediata quando o risco de morte é iminente, requerendo equipes preparadas. (MENDES,2018)

Para tanto, há que se formar os profissionais com as competências necessárias para atender as demandas que os serviços requerem. Nas unidades de urgência e emergência relevam-se os conhecimentos, habilidades e atitudes específicas a fim de prestar assistência individualizada, digna e humanizada a quem busca por esse tipo de atendimento, que no caso da humanização, inclui acolhimento, comunicação, diálogo, resolutividade, respeito e saber ouvir. (HOLANDA, 2014)

Complementa, Oliveira (2014) que o acolhimento e a classificação de risco, são essenciais na prática de atendimento humano para que haja qualidade e priorização eficaz, sendo estas prerrogativas evidenciadas como função destinada ao profissional enfermeiro, pois este reúne todas as condições necessárias para a aplicação de escalas de avaliação e, posteriormente, realizar a classificação de risco, ordenando a ordem e a forma de atendimento.

A classificação de risco é uma área de atuação do enfermeiro que possibilita, além da experiência profissional, a padronização no atendimento com ampliação da qualidade do serviço de enfermagem, aprimoramento da assistência e o gerenciamento da unidade de saúde. (FARIAS, 2017)

Segundo Brasil (2009), Todos os profissionais de saúde devem realizar o acolhimento do paciente e sua família, mas cabe ao enfermeiro a atividade de classificação de risco do paciente.

A classificação de risco de urgência é, portanto, uma ferramenta que além de organizar a fila de espera e propor outra ordem de atendimento que não a ordem de chegada, tem também outros objetivos importantes, como: garantir o atendimento imediato do usuário com grau de risco elevado; promover o trabalho em equipe por meio da avaliação contínua do processo; dar melhores condições de trabalho para os profissionais; aumentar a satisfação dos usuários e, principalmente, possibilitar e instigar a pactuação e a construção de redes internas e externas de atendimento. (SANTOS, 2014)

Assim, quando o paciente procura o serviço de saúde é acolhido pelo enfermeiro que realiza a escuta qualificada, avalia e aplica o fluxograma norteador e 
classifica as necessidades de saúde daquele, conforme critérios de risco estabelecidos em protocolos. (HERMIDA, 2018)

A inserção do enfermeiro durante e após a classificação de risco, exige escolhas e atitudes que reforcem sua autonomia profissional e habilidades pessoais, não se limitando ao registro e à classificação do usuário por meio de discriminadores. (CHAVES, 2014)

Tais ideias, discutidas pelos autores a respeito dessa temática, que coloca o enfermeiro como protagonista no acolhimento com classificação de risco, são baseadas na RESOLUÇÃ O COFEN N 661/202I. Segundo esta resolução, no âmbito da equipe de enfermagem a classificação de risco e priorização da assistência em serviços de urgências é privativa do enfermeiro. (COFEN, 202I)

\subsection{A Alta Demanda nos Serviços de Urgência e Emergência}

O sistema de saúde brasileiro está estruturado em níveis de baixa (atenção primária), média (atenção secundária) e alta densidades tecnológicas (atenção terciária), visando garantir a integralidade do cuidado. A porta de entrada do usuário no sistema é a atenção primária, estruturada como primeiro nível de atenção para garantir atendimento das necessidades de saúde de toda a população. (MINISTÉRIO DA SAÚDE, 2010)

Entretanto, devido à falta de agilidade e baixa resolutividade dos serviços de atenção básica, a população com menor renda demonstra baixa adesão aos serviços oferecidos nesse nível de atenção, preferindo usar atendimento para suas necessidades de saúde nos Serviços de Urgência e Emergência (SUE), como alternativa para conseguir atendimento em menor prazo. (AZEVEDO, 2010)

A busca constante pelos serviços de emergência por casos de menor gravidade pode indicar tanto obstáculos na utilização da rede de atenção à saúde quanto a vulnerabilidade de pessoas que necessitam de cuidados repetidamente. (ACOSTA, 2015)

O desconhecimento da população frente à oferta de serviços de saúde ou a utilização inadequada dos mesmos faz com que a classificação de risco se torne ineficiente em alguns aspectos, já que o atendimento prestado aos usuários 
classificados como menos graves se torna superficial e inadequado. (NASCIMENTO, 20II)

A maioria desses serviços funciona acima de sua capacidade instalada, com profissionais sem capacitação, gerenciamento inefetivo, ausência de planejamento, excesso de demanda e demanda inadequada (O'DWYER; OLIVEIRA; SETA, 2009)

De acordo com Garlet et al. (2009), o crescente aumento do atendimento no Serviço Hospitalar de Urgência relacionado ao aumento dos acidentes e da violência urbana, às questões socioeconômicas, à falta de leitos para internação na rede pública, ao aumento da longevidade da população, assim como à falta de agilidade e de resolutividade dos serviços de saúde.

A crise no Serviço Hospitalar de Urgência é agravada, também, pela procura por pessoas em situação de urgência, juntamente com aquelas em condições de saúde não graves, dificultando o estabelecimento de prioridades. (MARQUES; LIMA, 2008)

A identificação da demanda de acesso dos usuários aos serviços de saúde é fundamental no gerenciamento da capacidade de utilização dos recursos disponíveis, assim como a superlotação nos serviços de emergência com problemas de saúde não urgentes tem atraído atenção mundial e motivado vários estudos nesta área (VAN UDEN et al., 2005).

Estudos realizados em diferentes países mostram que a implantação de protocolos de triagens nos serviços de emergência permitiu que os casos considerados de maior gravidade fossem priorizados, com impacto nas taxas de mortalidade. (LUNET; PINTO; AZEVEDO, 2010)

A superlotação é o retrato do desequilíbrio entre a oferta e a procura, que pode ser agravada por problemas organizacionais, como o atendimento sem estabelecimento de critérios clínicos, o que pode acarretar graves prejuízos aos pacientes. O excesso de triagem não só desperdiça recursos como causa atraso de tratamento para os casos mais graves. (SOUZA, 2015) 


\subsection{A importância do Acolhimento com Classificação de Risco como uma ferramenta (protocolo) essencial na Urgência e Emergência}

Reconhece-se que, a priorização de pacientes graves pode viabilizar o manejo da doença no tempo adequado, com maiores chances de recuperação de casos agudos atendidos em Serviço Hospitalar de Emergência. (FITZGERALD, 2010)

Para isso, foram desenvolvidos sistemas de classificação de risco em diversos países, dentre os quais se destacam: National Triage Scale (NTS) da Austrália; Canadian Emergency Department Triage and Acuity Scale (CTAS) do Canadá; Manchester Triage System (MTS) do Reino Unido; e Emergency Severity Index (ESI) dos Estados Unidos da América. (GANLEY, 20II)

No Brasil, desde 2004, tem sido recomendado por órgão governamental, o Acolhimento com Classificação de Risco. (INOUE; JUNIOR, 2015)

O Acolhimento com classificação de risco é um sistema que transcende a lógica perversa de atendimento por ordem de chegada e se diferencia de outros sistemas de classificação de risco por se fundamentar no fortalecimento de vínculo entre usuário e trabalhadores, por meio da escuta qualificada. Artigo 26

A saber, o Acolhimento com Classificação de Risco consiste na sistematização de um processo de Classificação de Risco, baseado em quatro níveis de gravidade, com ênfase nas ações de acolhimento do paciente e do seu acompanhante pela equipe de saúde, para a humanização da assistência. (INOUE; JUNIOR, 2015)

Independentemente do sistema, a classificação de risco é um importante aspecto dos cuidados à saúde. (GANLEY, 20II)

Isso porque, quando realizada por enfermeiros experientes, além de contribuir à segurança do paciente, oportuniza a racionalização dos recursos da atenção à saúde. (SVEDLUND, 2014)

Os protocolos são ferramentas úteis; porém, se utilizadas de forma isolada, não são suficientes, pois não envolvem outros fatores como aspectos culturais e sociais que podem ser o ponto fundamental para uma adequada avaliação do risco de cada pessoa. (SOUZA; TOLEDO, 20II) 
As triagens realizadas nos serviços de urgência sem a utilização de protocolos eram até pouco tempo atrás feitas de forma intuitiva e sem utilizar uma metodologia específica. Desta forma, não eram replicáveis aos outros profissionais de saúde e nem serviam de parâmetro para auditorias. (MACKWAY-JONES; MARSDEN, 2010)

$\mathrm{O}$ acolhimento do paciente nos serviços de emergência deve ocorrer mediante a utilização de protocolos que estratifiquem e classifiquem o risco apresentado, buscando a priorização do atendimento de acordo com a gravidade apresentada pelo caso. (AMTHAUER; CUNHA, 20I6)

Nesse sentido, embasando-se na Política Nacional de Humanização (PNH), o Ministério da Saúde propôs a implantação do acolhimento com classificação de risco (ACCR), sendo o enfermeiro responsável pela avaliação do paciente. (MINISTÉRIO DA SAÚDE, 20I5)

O ACCR permite a garantia da humanização na assistência, a acessibilidade e a realização de um atendimento mais digno e menos excludente ao usuário e seus familiares. (BELLUCCI JÚNIOR; VITURI, 2015)

\section{CONCLUSÃO}

Ao longo do trabalho verificou-se a importância do enfermeiro como o referencial na aplicação dos protocolos da classificação de risco, sendo esse profissional qualificado para direcionar e dimensionar o risco em que está o paciente.

Para que a classificação de risco possa de fato cumprir sua função, é relevante que haja divulgação para população em geral sobre as vantagens da utilização de protocolos direcionados a classificação de risco. Pois no entendimento popular, o conceito de atendimento de urgência encontra-se entrelaçado à rapidez na prestação da assistência, sendo esta compreensão prejudicial para o estabelecimento de critérios de definição de prioridades no atendimento. Essa problemática tende a culminar na superlotação dos centros de atendimento de urgência e emergência, uma vez que, a atenção primária a saúde, acaba não conseguindo suprir as demandas as necessidades de saúde de toda a população. 
O enfermeiro deve conhecer as disponibilidades do serviço de saúde do seu município, obtendo assim, a capacidade de direcionar o atendimento do serviço especializado. Assim, permite caracterizar o fluxo dos usuários no sistema de saúde.

A classificação de risco é um instrumento que busca a minimização dos agravos a saúde. Os artigos analisados indicam que a classificação de risco melhora o fluxo de pacientes atendidos na emergência e proporciona maior resolutividade nas respostas ao usuário.

Conclui-se que o enfermeiro pode contribuir para um atendimento mais equânime da saúde pública, à medida em que busca por preparo técnico e científico para o desempenho de ações e realiza planejamentos que visem otimização dos recursos da saúde e o aumento da satisfação dos trabalhadores e usuários do sistema público.

\section{REFERÊNCIAS}

BELLUCCI JÚNIOR, J. A. B.; MATSUDA, L.M. Construction and validation of an instrument to assess the Reception with Risk Rating. Revista Brasileira de Enfermagem, Brasília, DF, n. 65, v. 5, p. 751-757, set./out., 2012.

ULHÔA, Maria de Lourdes; GARCIA, Fernando Coutinho; LIMA, Cristina Torres; SANTOS, Daniela Soares; CASTRO, Pedro Adalberto Aguiar. A IMPLANTAÇÃO DE NOVA TECNOLOGIA: IMPLICAÇÃO NA EFICIÊNCIA DO TRABALHO NA UNIDADE DE PRONTO ATENDIMENTO DE UM HOSPITAL PÚBLICO DE URGÊNCIA E EMERGÊNCIA

BRASIL. Ministério da Saúde. Secretaria de Atenção à Saúde. Acolhimento e Classificação de Risco nos serviços de urgência. Brasília, DF, 2009.

DAL PAI, Daiane; Lautert, Liana. Sofrimento no trabalho de enfermagem: reflexos do "discurso vazio" no acolhimento com classificação de risco. Esc. Anna Nery, Rio de Janeiro, v. 15, n. 3, p. 524-530, Sept. 2011 .

MARIA, Monica Antonio; QUADROS, Fátima Alice Aguiar; GRASSI, Maria de Fátima Oliveira. Sistematização da assistência de enfermagem em serviços de 
urgência e emergência: viabilidade de implantação. Rev. bras. enferm. Brasília, v. 65, n. 2, p. 297-303, Apr. 2012 .

NASCIMENTO, E. R. P. do, Hilsendeger, B. R., Neth, C., Belaver, G. M., \& Bertoncello, K. C. G. Acolhimento com classificação de risco: avaliação dos profissionais de enfermagem de um serviço de emergência. Revista Eletrônica De Enfermagem, 2011. 13(4), 597-603.

OLIVEIRA, Acolhimento com classificação de risco: Percepções de usuários de uma unidade de pronto atendimento.Texto Contexto Enferm, 2017; 26(I):eog6oor4

SAÚDE, Ministério da. PORTARIA № 354, DE ıo DE MARÇO DE 2014.

SAÚdE, Ministério da. ACOLHIMENTO NAS PRÁTICAS DE PRODUÇÃO DE SAÚDE. 2010

SANTOS, Aline Karen Nunes dos; SORATTO, Maria Tereza. Segurança do paciente nas Unidades de Urgência Emergência. 2018

SOUZA, Cristiane Chaves de; TADEU, Luiza Ferreira Ribeiro. CLASSIFICAÇÃO DE RISCO EM PRONTO SOCORRO: CONCORDANCIA ENTRE UM PROTOCOLO INSTITUCIONAL BRASILEIRO E MANCHESTER. 20Ir. 8 f. Dissertação (Mestrado) - Curso de Mestre em Enfermagem, Revista Latino-americana Enfermagem, Belo Horizonte, 2oro. Cap. I9.

VITURI, Dagmar Willamowius; INOUE, Kelly Cristina; BELLUCCI JÚNIOR, José Aparecido; OLIVEIRA, Carlos Aparecido de; ROSS, Robson Marcelo; MATSUDA, Laura Misue. Acolhimento com classificação de risco em hospitais de ensino: avaliação da estrutura, processo e resultado.

SIQUEIRAI, Carolina Poite de; FIGUEIREDO, Karla Crozeta; KHALAF, Daiana Kloh; WAL, Marilene Lowen; BARBOSA, Sayonara de Fátima Faria; POLI, Thaiane Almeida Silva. Segurança do paciente em uma unidade de pronto atendimento: planejamento de ações estratégicas. 
AZEVEDO, Jane Mary Rosa; BARBOSA, Maria Alves. TRIAGEM EM SERVIÇOS DE SAÚDE: PERCEPÇÕES DOS USUÁRIOS.

RONCALLI, Aline Alves; OLIVEIRA, Danielle Nogueira de; SILVA, Izabella Cristina Melo; BRITO, Robson Figueiredo; VIEGAS, Selma Maria da Fonseca. PROTOCOLO DE MANCHESTER E POPULAÇÃO USUÁRIA NA CLASSIFICAÇÃO DE RISCO: VISÃO DO ENFERMEIRO

GUEDES, Helisamara Mota; MARTINS, José Carlos Amado; CHIANCA, Tânia Couto Machado. Valor de predição do Sistema de Triagem de Manchester: avaliação dos desfechos clínicos de pacientes.

GOUVEIA, Mariana Tomé; MELO, Suélida Rafaela de; COSTA, Maria Wanessa da Silva; SOUZA, José Madson Medeiros; SÁ, Laisa Ribeiro de; PIMENTA, Cláudia Jeane Lopes; COSTA, Kátia Neyla de Freitas Macedo; COSTA, Tatiana Ferreira da. ANÁlisE DO ACOLHIMENTO COM CLASSIFICAÇÃO DE RISCO EM UNIDADES DE PRONTO-ATENDIMENTO.

SOUSA, Kayo Henrique Jardel Feitosa; DAMASCENO, Carolinne Kilcia Carvalho Sena; AlMEIDA, Camila Aparecida Pinheiro Landim; MAGALHÃES, Juliana Macêdo; FERREIRA, Márcia de Assunção. Humanização nos serviços de urgência e emergência: contribuições para o cuidado de enfermagem.

CAMARGO NETO, Orlando; ANDRADE, Gleice Kelli Santana de; KARPIUCK, Luciana Brondi; GANASSIN, Amanda Rodrigues. A Atuação do Enfermeiro no Sistema de Acolhimento e Classificação de Risco nos Serviços de Saúde.

DEUS, Gabriel Alves de; FERREIRA, Jader Henrique; MONTANDON, Diego Santiago; GODOY, Simone de. Acolhimento com avaliação e classificação de risco em um pronto socorro: estudo comparativo.

Hermida PMV, Nascimento ERP, Echevarría-Guanilo ME, Brüggemann OM, Malfussi LBH. User embracement with risk classification in an emergency care unit: an evaluative study. Rev Esc Enferm USP. 2018;52:e03318. 
Marques LA, César FCR, Izidoro LCR, Cabral KB, Santos LF, Brasil VV, et al. Satisfação de usuários com o acolhimento e classificação de risco em unidades públicas de saúde. Rev. Eletr. Enf. [Internet]. 2018.

Malfussi, Luciana Bihain Hagemann de et al. CONCORDÂNCIA DE UM PROTOCOLO INSTITUCIONAL DE AVALIAÇÃO COM CLASSIFICAÇÃO DE RISCOı I Manuscrito extraído de dissertação - Acurácia da classificação de risco por enfermeiros de uma unidade de emergência hospitalar, apresentado ao Programa de Mestrado Profissional Multidisciplinar em Saúde, da Universidade Federal de Santa Catarina (UFSC), em 2013. Texto \& Contexto - Enfermagem [online]. 2018, v. 27 , n. I.

Feijó, Vivian Biazon El Reda et al. Análise da demanda atendida em unidade de urgência com classificação de risco. Saúde em Debate [online]. 2015, v. 39, n. Io6.

Costa, Maria Antonia Ramos et al. Acolhimento com Classificação de Risco: Avaliação de Serviços Hospitalares de Emergência. Escola Anna Nery [online]. 2015, v. 19, n. 3 .

Inoue, Kelly Cristina et al. Avaliação da qualidade da Classificação de Risco nos Serviços de Emergência. Acta Paulista de Enfermagem [online]. 2015, v. 28, n. 5.

Mendes TJM, Silveira LM, Silva LP, Stabile AM. Association between reception with risk classification, clinical outcome and the Mews Score. REME Rev Min Enferm. 2018;22:e-1077. doi: https://doi.org/10.5935/1415-2762.20180007.

Holanda FL, Castagnari MC, Cunha ICKO. Construction of a Professional Competency Matrix of the nurse in emergency services. Acta Paul Enferm. 2014;27(4):373-9.

Oliveira GN, Campanharo CRV, Okuno MFP, Batista REA. Nursing care based on risk assessment and classification: agreement between nurses and the institutional protocol. Rev Latino Am Enferm 2013;21(2):500-6. 
Farias JC, Nebel CZ, Moraes CL, Casagranda LP, Lange C, Santos F. Acolhimento com avaliação e classificação de risco como ferramenta de gestão: percepção dos enfermeiros. Publ Biol 2017;22(I):40-7.

Brasil. Ministério da Saúde; Secretaria de Atenção à Saúde. Política Nacional de Humanização da Atenção e Gestão do SUS. Acolhimento e classificação de risco nos serviços de urgência. Brasília: MS; 2009.

Santos AP, Freitas P, Martins HM. Manchester Triage System version II and resource utilization in the emergency department. Emerg Med J. 2014;31(2):148-52.

Chaves CS, Santos DA, Teixeira Silva LL, Ferreira MLR, Chianca, TCM. Nurses' perception about risk classification in emergency services. Invest Educ Enferm [Internet]. 2014 [cited 2017 Jun 07]; 32(I):78-86.

Azevedo ALM, Costa AM. A estreita porta de entrada do Sistema Unico de Saude (SUS): uma avaliacao do acesso na Estrategia de Saude da Familia. Interface (Botucatu) [Internet]. 2010 [cited 2017 Aug. 16]; 14(35):797-810. Available from: http://www.scielo.br/pdf/icse/vi4n35/30ro.pdf.

Acosta AM, Lima MADS. Frequent users of emergency services: associated factors and reasons for seeking care. Rev Latino-am Enfermagem [Internet]. 2015 [cited 2016 Aug 9]; 23(2):337-44.

Nascimento ERP, Hilsendeger BR, Neth C, Belaver GM, Bertoncello KCG. Classificação de risco na emergência: avaliação da equipe de enfermagem. Rev Enferm UERJ [Internet]. 20II [cited 2015 Jul I5]; I9(I):84-8.

O’DWYER, G.; OlIVEIRA, S. P.; SETA, M. H. Avaliação dos serviços hospitalares de emergência do programa QualiSUS. Ciência \& Saúde Coletiva, Rio de Janeiro, n. I4, v. 5, p. I88I-90, 2009.

VALENTIM, M. R. S.; SANTOS, M. L. S. C. Políticas de saúde em emergência e a enfermagem. Revista Enfermagem UERJ, Rio de Janeiro, n. 17, v. 2, p. 285-289, 2009. Disponível em: . Acesso em: 20 abr. 2014. 
MARQUES, G. Q.; LIMA, M. A. D. S. Technological organization of labor in an emergency service and nursing worker's autonomy. Revista da Escola de Enfermagem da USP, São Paulo, n. 42, v. I, p. 41-47, 2008.

VAN UDEN, C. J. T. et al. The impact of a primary care physician cooperative on the caseload of an emergency department: the Maastricht integrated out-of- -hours service. Journal of General Internal Medicine, Philadelphia, v. 20, n. 7, p. 612-617, 2005 .

LUNET, N.; PINTO D.; AZEVEDO A. Sensitivity and specificity of the Manchester Triage System for patients with acute coronary syndrome. Rev Port Cardiol., Lisboa, n. 29, v. 6, p. 961-987, 2010.

Souza CC, Araújo FA, Chianca TCM. Scientific literature on the reliability and validity of the Manchester Triage System (MTS) Protocol: a integrative literature review. Rev esc enferm USP. [Internet]. 2015 Feb [cited 2016 Feb Io];49(I):I44-5I.

FitzGerald G, Jelinek GA, Scott D, Gerdtz MF. Emergency department triage revisited. Emerg Med J. 2010 Feb; 27(2):86-92.

Ganley L, Gloster AS. An overview of triage in the emergency department. Nurs Stand. 2011; 26(12):49-56.

Ek B, Svedlund M. Registered nurses' experiences of their decisionmaking at an Emergency Medical Dispatch Centre. J Clin Nurs. 2014 Oct 2. doi: Io.IIII/jocn.I270I.

Pai DD, Lautert L. Sofrimento no trabalho de enfermagem: reflexos do "discurso vazio" no acolhimento com classificação de risco. Esc Anna Nery. [Internet]. 20II [citado 2015 dez Io];15(3):524- 30.

Souza CC, Toledo AD, Tadeu LFR, Chianca TCM. Risk classification in an emergency room: agreement level between a Brazilian institutional and the Manchester Protocol. Rev Latino-Am Enfermagem. [Internet]. 2011 Feb [cited 2016 Feb 10]; 19(I):26-33. 
Mackway-Jones K, Marsden J, Windle J. Grupo Brasileiro de Classificação de risco. Sistema Manchester de Classificação de Risco. 2. ed. Belo Horizonte (MG): Grupo Brasileiro de Classificação de risco; 2010.

Amthauer C, Cunha MLC. Manchester Triage System: main flowcharts, discriminators and outcomes of a pediatric emergency care. Rev Latino-Am Enferm. 2016[citado em 2018 jul. 15];24:e2779.

Ministério da Saúde (BR). Política Nacional de Humanização - PNH. 2015 [citado em 2018 jul. 15]. Brasília: Ministério da Saúde; 2015.

Bellucci Júnior JA, Vituri DW, Versa GLGS, Furuya OS, Vidor RC, Matsuda LM. Hospital emergency service patient reception with triage: evaluation of the care process. Rev Enferm UERJ. 2015[citado em 2018 jul. 15];23(2):82- 7.

COFEN. RESOLUÇÃO COFEN N 66ı/202ı. Brasília, II de março, 202ı. 